\title{
Políticas europeas de la "hostipitalidad" en el cine de Ruben Östlund: el caso de The Square (2017)
}

\section{The European politics of "hostipitality" in the cinema of Ruben Östlund: The Square (2017) as a case-study}

\author{
Mónica Fernández Jiménez \\ Universidad de Valladolid, España \\ monica.fernandez@uva.es https://orcid.org/o0oo-0002-8988-6930
}

Sofía Martinicorena Zaratiegui

Universidad Complutense de Madrid, España smartini@ucm.es https://orcid.org/o0oo-0001-6605-7163

Inés Paris Arranz

Universidad Complutense de Madrid, España ipariso1@ucm.es https://orcid.org/oooo-0003-4585-2736

\begin{abstract}
Resumen:
El presente artículo analiza la última película del director sueco contemporáneo Ruben Östlund, The Square (2017), a través de la teoría de la hospitalidad. Frente al ideal de un supuesto "sueño europeo", en palabras de Jeremy Rifkin (2004), argumentaremos que la trayectoria fílmica de Östlund explora, desde una sensibilidad posmoderna, las contradicciones entre los discursos sobre la hospitalidad europea y su cristalización en los espacios del capitalismo tardío. En particular, exploraremos las constantes temáticas y técnicas de su producción en el contexto del cine europeo y sueco, entre las que destaca su particular visión del acercamiento al Otro y el análisis distanciado, casi antropológico, de la ética que hay en juego en los espacios público-privados de las sociedades occidentales contemporáneas. Tomaremos la película The Square como ejemplo de la imposibilidad de una ética hospitalaria, sugiriendo como conclusión que la realidad europea contemporánea se acerca más al concepto derridiano de la "hostipitalidad" que a un idealizado sueño europeo.
\end{abstract}

\begin{abstract}
:
This paper analyses The Square (2017), the last film by contemporary Swedish director Ruben Östlund, through the lens of hospitality theory. Working against the ideal of an alleged "European dream", as per Jeremy Rifkin (2004), we will argue that Östlund's cinema explores the contradictions between the discourses on European hospitality and their materialisation in the spaces of late capitalism. In particular, we will explore the thematic and technical landmarks of his production within the context of Swedish and European cinema, namely, his personal take on the approach to the Other and his detached, almost anthropological analysis of the ethics at stake within the public and private spaces of contemporary Western societies. The Square will be taken as an example of the impossibility of an ethics of hospitality, suggesting as a conclusion that the contemporary European reality has more in common with the Derridean idea of "hostipitality" than with an idealised European dream.
\end{abstract}

Palabras clave: Ruben Östlund; cine europeo; posmodernidad; espacio; hospitalidad; excepcionalismo.

Keywords: Ruben Östlund; European cinema; postmodernity; space; hospitality; exceptionalism. 


\section{Introducción ${ }^{*}$}

Los versos de Emma Lazarus grabados sobre la base de la Estatua de Libertad -“Give me your tired, your poor, / Your huddled masses yearning to breathe free"- son ya ampliamente reconocidos como uno de los símbolos más contradictorios de la sociedad estadounidense. Un hecho más visiblemente evidenciado en el presente con los discursos xenófobos de Donald Trump, los Estados Unidos nunca fueron un paraíso para sus Otros. Desde la masacre de numerosos nativos americanos antes de que este territorio fuese un país llegando hasta el imperialismo global en su paso por la esclavitud institucionalizada, el excepcionalismo americano se ha convertido en una doctrina a deconstruir en el ámbito de los estudios culturales. A ello se dedican en parte Ana $\mathrm{M}^{\mathrm{a}}$ Manzanas Calvo y Jesús Benito Sánchez en su libro Hospitality in American Literature and Culture (2017). Este monográfico, en sus propias palabras, "se centra en las maneras en las que los Estados Unidos administran protocolos de pertenencia y nopertenencia y distingue entre aquellos que se pueden sentir en casa y aquellos que siempre estarán fuera del cuerpo político pese a haber sido los 'huéspedes' originales” (traducción propia; 2017, n.p.). De este modo, la teoría sobre la hospitalidad y sus ineludibles condiciones supone un freno a todo discurso excepcionalista que enmascare las complejidades a las que se enfrentan nuestras sociedades occidentales contemporáneas.

Que Jeremy Rifkin afirme en su libro The European Dream que el sueño americano está siendo sustituido por un sueño europeo (2004, p. xii) nos hace preguntarnos si este moralmente dudoso excepcionalismo americano no estará siendo imitado por los poderes existentes en Europa. De hecho, a un nivel más local, Helena Karlsson identifica un denominado "excepcionalismo sueco" que presupone que dicha nación es la más antirracista de todos los países occidentales (2014, p. 44). Pero, pese a las alabanzas de Rifkin estableciendo que el sueño europeo difiere del americano en su sentido más amplio de comunidad y bienestar social (2004, p. xii), eventos recientes

\footnotetext{
* La investigación necesaria para la redacción de este artículo ha sido financiada gracias al proyecto ERASMUS+ (European Project 2017-1-ESo1-KA203-038181).
} 
evidencian que dichos excepcionalismos son propensos a ser igualmente cuestionados: los retos que supone la actual crisis migratoria y de refugiados en Europa así como el creciente auge de discursos e ideologías xenófobas en estos países han hecho resquebrajarse los valores que llevaron a adoptar el Sistema Europeo Común de Asilo (SECA) en la Convención de Ginebra de 1951 (Hospitality n.p.). Ha sido a través del cine europeo que se ha comenzado a mostrar la doble cara de esta visión positiva, de la mano de directores como Ken Loach, Pawel Pawlikowski o Aki Kaurismäki entre otros. Estas películas también evidencian lo que Hugo Münstenberg ya plasmó en 1916 con la publicación de The Photoplay: A Psychological Study: que hay una relación especial entre la psicología del espectador y lo que se muestra en la pantalla (Moure, 2011, p. 25). Es así que el cine consigue un mayor efecto que otros géneros para "evaluar la visibilidad/invisibilidad del migrante, su in/audibilidad, así como las técnicas de vigilancia que son parte de la hostipitalidad que le espera al Otro extranjero" (traducción propia; Hospitality, n.p.).

Desde una sensibilidad específicamente posmoderna, uno de los cineastas que más recientemente ha explorado este asunto es Ruben Östlund, el último sueco en ganar la Palma de Oro del Festival de Cannes desde Alf Sjöberg en 1951 con su película The Square (2017). El presente artículo analiza cómo The Square, la última película de este director y probablemente la más conocida internacionalmente, explora las dimensiones espaciales de la hospitalidad en su manera de operar en los no-lugares de la sociedad contemporánea sueca y, por extensión, europea. Comenzaremos con una introducción que sitúa al cine de Östlund en el contexto europeo del que nace y se nutre, e identificaremos los hitos de su muy particular lenguaje cinematográfico a fin de comprender cómo la técnica audiovisual de Östlund contribuye a la exploración de los problemas presentes a lo largo de su filmografía. Argumentaremos que dichos problemas y preocupaciones culminan en The Square, destacando entre ellos la preocupación por los espacios urbanos, el ambiguo encuentro con el Otro, y, sobre todo, la crítica a 
una imagen autocomplaciente de una Europa con unos estándares éticos cuestionables.

\section{Europa frente a Europa: la visión posmoderna de Ruben Östlund}

Ruben Östlund pertenece a una joven generación de cineastas europeos cuyas preocupaciones, tanto técnicas como temáticas, suponen un desafío a las convenciones fílmicas y culturales y aportan perspectivas innovadoras y críticas en su retrato de una sociedad europea a menudo autocomplaciente y que niega sus propios conflictos internos (Karlsson, 2014, p.44). Los pilares de esta sociedad ya han sido abordados de manera mordaz por directores europeos de la misma generación de Östlund. Películas como Canino (2009), de Yorgos Lanthimos-una sátira sobre la institución de la familia y el tabú del incesto-o Toni Erdmann (2016), de Maren Ade-que con humor denuncia el crecimiento de la superficialidad en la era del neoliberalismo en Europa-son ejemplos de una generación de cineastas que recogen la herencia posmoderna y trascienden el neorrealismo que dominó la época de posguerra en Europa (Åberg, 2010, p. 252) y que, sin embargo, entablan de manera compleja un diálogo sobre la sociedad que representan. ${ }^{1}$

Si bien el cine crítico con una Europa antirracista y hospitalaria idealizada no es novedoso, Östlund aporta un punto de vista nuevo. Mientras que típicamente el cine de crítica social se ha esforzado en proveer la narrativa del migrante y su punto de vista, Östlund narra el encuentro con el Otro desde el punto de vista del "huésped” o “anfitrión”. El director sueco, por tanto, encaja en la tradición señalada por Karlsson según la cual en Suecia las voces de las personas no blancas son minoritarias en el cine, tanto en forma

\footnotetext{
${ }^{1}$ Quizá uno de los esfuerzos más célebres y autoconscientes de inaugurar un nuevo cine en estas líneas fuera el controvertido movimiento Dogma 95, liderado por Lars von Trier y al que se han unido importantes figuras como Susanne Bier, con el fin de deshacerse de ciertas convenciones del lenguaje cinematográfico tradicional y de inaugurar una nueva manera de hacer cine (Lindqvist, 2016, p. 550). Si bien los esfuerzos del danés no guardan una relación directa con el director que nos ocupa, sí que apuntan hacia Europa, y en particular a los países del entorno nórdico, como productores de un cine vanguardista y rompedor.
} 
de personajes, normalmente estereotipados, como en lo que a cineastas se refiere (2014, p. 48). No queriendo apropiarse de la voz de los Otros de su sociedad, Östlund pone el foco en las sociedades de consumo que describieron Fredric Jameson y Jean Baudrillard ${ }^{2}$ y que tuvieron como consecuencia ciertas transformaciones de los espacios e incluso en la percepción de la realidad. De este modo, Östlund consigue establecer una conexión más profunda entre el capitalismo tardío y la creciente hipocresía, más que xenofobia, que define a sus personajes europeos, explorada haciendo uso notable del humor y la ironía. Así, Östlund refleja esta tradición condescendiente en lo que Karlsson denomina el excepcionalismo sueco (2014, p. 44), que ignora la tendencia segregacionista de su sociedad y la ceguera de la clase acomodada. A esto se refiere Karlsson como "odio posmoderno hacia el Otro" (traducción propia; 2014, p. 46), cuya característica principal es que se mantiene inexplorado por el perpetrador, quien promulga una suerte de elitismo moral (ibid). Así se entiende que Christian, el protagonista de la película más aclamada de Östlund, The Square, no sea uno de los muchos "Otros" que desfilan por el film sino un hombre privilegiado que se autoproclama humanista, al que el director desafía poniéndole obstáculos que supongan una ruptura o cuestionamiento de su proyecto de identidad moral, como explica el propio cineasta en una entrevista para Build Series (BUILD Series 2018, 8:00- 8:30). Östlund consigue así reflejar una de las preocupaciones del posmodernismo, a saber, la inaccesibilidad a una realidad que se halla escondida bajo la forma del simulacro. Son las representaciones, los símbolos, los discursos (como los de Christian) los que engendran la realidad (Baudrillard, 1994, p. 1-2). Östlund

\footnotetext{
2 La sociedad de consumo ha sido descrita por diversos teóricos de la era del posmodernismo. Uno de ellos, Frederic Jameson, sitúa esta época tras la segunda guerra mundial (1998, p. 656), cuando el capitalismo más individualista con su fe en los logros del sujeto y sus capacidades es sustituido por corporaciones y multinacionales (1998, p. 658). Jean Baudrillard, para describir la consecuente indeterminación de las funciones en estas sociedades da el ejemplo del "hipermercado" como institución y lugar alrededor del cual se desarrolla el estilo de vida posmoderno; se trata de la articulación de la vida de acuerdo a nolugares, como diría Marc Augé, sin funciones específicas y cuyas dinámicas de hospitalidad se rigen por la capacidad de consumo (Baudrillard 1994, p. 77-78; 2009, p. 40).
} 
pone de relieve, precisamente, la propia artificialidad del simulacro en que viven personajes como Christian, convencidos de su impecable humanismo.

Esta perspectiva ha de entenderse en el contexto particular de las preocupaciones que atraviesan los preceptos de Escuela de Cine de Gotemburgo, donde Östlund estudió y con la cual mantiene una estrecha relación (Söderbergh Widding, 2016, p. 120). El desarrollo y reciente relevancia de la Escuela han supuesto un punto de inflexión en el cine sueco, anteriormente relegado a Estocolmo (Söderbergh Widding, 2016, p.119) y capitalizado por figuras como la de Bergman. Esta escuela se identifica con las películas “de la nueva realidad” (traducción propia; Söderbergh Widding, 2016, p. 119) y destaca por su interés en las nuevas tecnologías y mediosparte esencial según Baudrillard y Jameson de los anteriormente mencionados simulacros por su papel en la destrucción y creación de la información de dicha nueva realidad (Baudrillard, 1994, p. 79; Jameson, 2002, p. 298-9). Curiosamente, Östlund, para quien las nuevas tecnologías suponen desde el inicio de su carrera un estímulo indispensable en su creación fílmica, participó como parte de un proyecto de esta escuela en la realización de la primera película grabada exclusivamente con un móvil (Söderbergh Widding, 2016, p.119). No es casual, por tanto, que el director trabaje exclusivamente en formato digital (Westerståhl Stenport y Traylor, 2014, p. 77) Asimismo, la Escuela de Gotemburgo otorga el mismo valor a la ética que a la estética, ofreciendo una formación marcadamente humanista a sus alumnos (Söderbergh Widding, 2016, pp. 118-121). Söderbergh Widding piensa que el carácter de las películas de Östlund encaja perfectamente con la filosofía de la escuela en su convicción en la necesidad de que el cineasta tenga el valor de indagar en los aspectos más incómodos de su sociedad y que, al reflejarlos y explorarlos, pueda así cambiarla (2016, p. 120). Además, la presencia de pantallas en todas sus películas, además de una multiplicidad de perspectivas, reinterpretan el espacio fílmico de manera metaficcional y posmoderna, desafiando la idea del encuadre único: las cámaras que han pasado a formar parte del día a día de nuestra sociedad son otro espacio 
narrativo dentro de la propia narrativa que supone el género fílmico. Así, se trasciende la idea de realismo en el cine y la de realidad única en la sociedad.

Más allá del contexto general de Gotemburgo, la crítica ha identificado a Östlund como un representante del "Nordic slow cinema", cuyas características incluyen "contención emocional, falta de resolución dramática, un ritmo letárgico, una crítica social implícita y una invitación al espectador a ser partícipe activo mediante la no manipulación de su respuesta” (traducción propia; Lindqvist 2016, p. 558). Aquí Östlund deja patente, como ha expresado en repetidas ocasiones (Lindqvist 2016, p. 549), la influencia de su maestro Roy Andersson, con quien comparte el uso de la cámara estática, situada a cierta distancia de los personajes, el tempo letárgico, y en general el interés por la condición humana (Lindqvist 2016, p. 549). Además, Östlund en prácticamente todo su cine emplea a lo que Roger Edholm se refiere como "flaunt[ing the film's] structure" (2018, p. 119), una técnica metaficcional que lleva al espectador a prestar especial atención a las estructuras convencionales del género fílmico y a detenerse en las propias convenciones del medio. Así, Östlund se posiciona como un cineasta posmoderno también al cuestionar las estructuras rígidas de cualquier institución, ya sea la narratología fílmica tradicional, la familia nuclear o los discursos sobre una supuesta Europa de acogida. Esta actitud está en consonancia con la característica desconfianza hacia las "grandes narrativas" con la que Jean François Lyotard también identifica el posmodernismo (1985, p. xxiv). Como ya se ha sugerido, los espacios en los que se desarrollan la mayoría de sus películas son esenciales para representar la lógica posmodernista en los funcionamientos del capitalismo del consumidor (véase el centro comercial en Play). Estos espacios o no-lugares responden a una hiperrealidad afuncional que a su vez refleja los logros democráticos de la clase media en términos de consumo (Baudrillard, 1994, p. 40; pp. 77-8; Dobson, 2017, n.p.) y que está carente de referentes históricos e identitarios porque en ellos solo domina lo urgente (Augé 1995, p.105). La tendencia a la segregación que estos espacios suponen -la ya más que obvia ghetoización de la Europa contemporánea- es algo que Östlund enfatiza en sus películas. 
Teniendo en cuenta este contexto cultural, no sorprende que las características técnicas y escénicas se entrelacen de manera indisociable con la crítica social presente en las películas de Östlund: en ellas, la cámara está a disposición del transcurrir de unas vidas europeas a menudo rotas, hipócritas y a veces absurdas, para las cuales, a pesar de todo, el director se reserva cierta empatía. El personal lenguaje fílmico que emplea Östlund responde así no tanto a criterios argumentales o narrativos sino más bien a una curiosidad antropológica (BUILD Series 2018, 30:40) que ponga el foco sobre las fallas del comportamiento humano. 3 En particular, Östlund ha declarado que está interesado en lo que se conoce como "bystander effect" o efecto espectador (BUILD Series 2018, 4:50), un fenómeno social que dicta que las personas responden a una situación de emergencia de manera más urgente cuando están en soledad que cuando están rodeadas de otras personas. Esta preocupación es un leitmotiv en toda su filmografía: ya en su primer largometraje, The Guitar Mongoloid (2004), presenciamos cómo la dueña de una cafetería le roba la guitarra a un niño que está tocando y pidiendo dinero cerca de su establecimiento porque le molesta y perjudica a su negocio sin que nadie alrededor parezca sentirse impelido a actuar e impedir la violencia de la escena. Igualmente, tanto en Involuntary (2008) como en Play (2011) presenciamos escenas en autobuses públicos donde los pasajeros ignoran el conflicto que, igual de incómodos que los espectadores, están presenciando. El ejemplo más paradigmático de este efecto lo encontramos en la performance del hombre-mono de The Square, donde los elegantes invitados a un cóctel del museo miran impasibles cómo aquel acosa a diversos asistentes, haciendo culminar en este episodio las escenas precedentes en la película en que, mientras suena música clásica extradiegética, los mendigos situados en el centro del encuadre son ignorados por los transeúntes cuyos pies son lo único que se ve de ellos. El impacto de estas escenas a menudo se consigue mediante una cámara fija grabando un plano amplio que cumple

\footnotetext{
3 Es esta vocación casi antropológica la que hace que el cine de Östlund mantenga cierta tensión con el género documental, al que a menudo se aproxima. No en vano, Östlund dirigió dos documentales antes de debutar con su primer largometraje, The Guitar Mongoloid, el cual fue acusado, precisamente por sus características híbridas, de no ser una película (Smallwood, 2015, n.p.).
} 
una función testimonial y que registra los movimientos, acciones y comportamientos de los personajes dejando al público espectador con la responsabilidad acerca de qué hacer, o a qué conclusiones llegar, con eso que está viendo en la pantalla.

Ante esta descripción, podría pensarse que el cine que describimos es sesudo, severo y más bien pesimista. Esta idea es, de hecho, un estereotipo sobre el cine sueco (Hjort y Lindqvist, 2016, p. 2). Lo cierto es, sin embargo, que el humor es un rasgo esencial del cine no solo de Östlund sino del cine de autor nórdico, en el cual el humor "abarca desde lo absurdo a lo oscuramente satírico, y normalmente sirve como instrumento de crítica social" (traducción propia; Lindqvist, 2016, p. 560). No es aventurado afirmar que es en The Square, una película de indudable carácter humorístico, a veces cercano al absurdo, donde Östlund alcanza su apogeo técnico y temático, encapsulando las preocupaciones que le vienen acompañando a lo largo de su filmografía de manera consistente y poniendo a disposición del espectador un riquísimo repertorio de técnicas audiovisuales que le interpelan de manera ineludible. Analizaremos ahora cómo en la exploración de estos asuntos que caracterizan a Östlund, la hospitalidad -o su imposibilidad- es una preocupación constante que se refleja en su lenguaje cinematográfico. Es por ello que la teoría de la hospitalidad que se aborda en el libro de Manzanas Calvo y Benito Sánchez, que destaca el trabajo de filósofos como Immanuel Kant o Jacques Derrida, resulta prolífica para delinear una relación entre la técnica y el contenido de la producción de Östlund, ambos a disposición del retrato de la identidad de una Europa contemporánea y cambiante.

\section{The Square, o la "hostipitalidad" europea}

La película The Square tiene como protagonista a Christian (Claes Bang), director de un reputado museo de arte contemporáneo en Estocolmo. El desencadenante de la acción es el momento en que Christian se da cuenta de que su teléfono móvil ha desaparecido, el cual tratará de recuperar a lo largo de la película. Su dificultad para lidiar con las sospechas acerca de los autores 
del hurto, así como las contradicciones éticas en las que incurre -de las cuales es plenamente consciente- desatan reflexiones que conciernen a la responsabilidad individual y colectiva en las sociedades europeas contemporáneas, así como las sospechas hacia todo aquello que se codifica como Otro. La estructura de la película, de manera muy similar a Fuerza Mayor, como explica Roger Edholm, hace uso de la convencional narrativa de orden-caos-vuelta al orden, la cual termina siendo irónica por su propia artificialidad (2018, p, 127). Dicha artificialidad está presente en el propio personaje de Christian, quien también construye otra narrativa tanto sobre sí mismo como sobre su sociedad sueca, autoconvenciéndose de sus valores humanistas.

Christian es un personaje complejo que de cierta manera representa las contradicciones de una Europa pagada de sí misma a la par que insegura de su propio estatus. Ejemplo de ello son los primeros minutos de la película. La incomodidad que reinará durante toda ella queda establecida desde estas primeras escenas, cuya cinematografía no hace sino subrayar de manera irónica la pretensión de Christian y de la vida que lo rodea. Cuando lo vemos por primera vez, justo después de unos créditos acompañados de música diegética que sugiere un escenario de fiesta, este está dormido en un sillón con la ropa del día anterior. La siguiente escena, en la que Christian es entrevistado por una periodista estadounidense (Elisabeth Moss), responde al tipo de plano que se ha convertido un rasgo de autor de Östlund: el plano general grabado con cámara estática hace la función, según Edholm, de mostrar lo que queda fuera de las narrativas que los personajes construyen sobre sí mismos (2018, p. 127). Así, hay una cámara que graba el encuadre -un concepto también entendido como metáfora de la narrativa autocomplaciente que los personajes crean alrededor de sí mismos (Edholm, p. 2018, 199-21), lo que también es un simulacro- de Christian y otra, la del cineasta/antropólogo, que nos muestra toda la realidad: que se acaba de despertar y una trabajadora tiene que colocar su traje aún arrugado para que dé la impresión deseada de director formal y culto de un museo de arte 
moderno. En este plano, un letrero de neón cortado parcialmente por la cámara reza en el fondo: "YOU HAVE NOTHING" ("no tienes nada”).

La película gira en torno al Cuadrado que le da nombre. Aquí, la dialéctica espacial de interiores/exteriores que se da en Play -donde los Otros, niños migrantes árabes y negros están relegados a los espacios exteriores- se retoma en otros términos, a saber, las ideas de hospitalidad que encontramos dentro del Cuadrado frente a aquellas que existen fuera del Cuadrado. Dicho Cuadrado es una instalación artística que el museo sueco se congratula de exhibir, y que consiste en, literalmente, un simple cuadrado dibujado en el suelo del espacio exterior del museo, donde aparece inscrito: "El cuadrado es un santuario de confianza y cuidados. Dentro de él, todos compartimos los mismos derechos y obligaciones”. El Cuadrado se presenta como un espacio ideal a la par que real (esto es, físicamente realizado) que delimita los valores de un código ético aparentemente distinto al que rige en su exterior. Lo cierto es que esta exhibición fue real:4 el propio Östlund, junto con su productor, preparó esta instalación para un museo sueco con el ánimo de, en sus propias palabras, "crear un espacio simbólico -un Cuadrado- dedicado a valores altruistas. Una suerte de santuario" (traducción propia; Allard y Ciment, 2017, p. 11). En un giro interesante de los acontecimientos, la placa del Cuadrado fue robada la misma noche en que se inauguró la instalación, y el Cuadrado fue tomado posteriormente por "músicos callejeros, mendigos y manifestantes" (traducción propia; 2018, Brooks, n.p.). Östlund aprovechó las diversas reacciones que suscitó su instalación a la hora de construir The Square, ratificando una vez más su interés por nutrirse de materiales eclécticos para construir sus producciones.

La representación física de un Cuadrado cuyos límites señalan la existencia de un código ético particular apunta a una de las preocupaciones constantes en la filmografía de Östlund, a saber, la delimitación de los espacios compartidos, públicos y privados, y su relación con el acuerdo social subyacente y con las nociones de responsabilidad derivadas de dichas

$4 \underline{\mathrm{http}: / / \text { vandalorum.se/en/square-ruben-\%C3\%B6stlund-kalle-boman }}$ 
demarcaciones (BUILD Series 2018). En particular, Östlund recuerda en la entrevista cómo su preocupación por las fronteras se vio reflejada en la creación del primer vecindario vallado ("gated community”) que se construyó en Suecia, lo cual le resultó impactante por ser una manera agresiva de establecer los límites a la responsabilidad y de declarar una amenaza todo aquello que quede más allá de la frontera del espacio. (BUILD Series 2018, 21:00). Tales preocupaciones toman una nueva relevancia en el contexto de un país como Suecia, una nación de supuesta reputación humanitaria y de buena acogida al vecino. Preguntado por ello en una entrevista en relación con su motivación tras la idea del Cuadrado en The Square, Östlund comenta que a partir de los años 80 tuvo lugar una deriva, tras el periodo fuerte de la socialdemocracia, en que según él han surgido dificultades sociales relacionadas con los servicios del estado del bienestar (Allard y Ciment, 2017, p. 12). Esta idea inmediatamente introduce la cuestión de la hospitalidad en nuestras sociedades. Para empezar, la respuesta de Östlund nos sugiere que el concepto de hospitalidad no es estático sino móvil, voluble y sensible a los cambios histórico-políticos. La hospitalidad no significa lo mismo ahora, en nuestro contexto social particular, que en otro cualquiera. Como afirman Manzanas Calvo y Benito Sánchez, "la hospitalidad ha cambiado de un discurso de generosidad a un discurso de espacialidad y desplazamiento; de una moral interpersonal a un asunto de política nacional" (traducción propia, 2017, p. 19), especialmente en el marco de influencia determinante que tienen las naciones-estado sobre sus ciudadanos (y, de manera crucial, sobre sus no-ciudadanos).

Ante ello cabe preguntarse: ¿cuál es la idea de hospitalidad que nos sugiere The Square? ¿Qué implicaciones tiene el Cuadrado, más allá de su inscripción? La película nos ofrece dos versiones de la hospitalidad: la ideal, correspondiente al Cuadrado, que aparentemente es incondicional, y la correspondiente a la vida cotidiana de los personajes de la película, claramente condicionada. Concluiremos que el desfase de ambos discursos -paralelos y contradictorios- sobre la hospitalidad no es sino una representación de esa hospitalidad aporética que Derrida sugiere con su término "hostipitalidad". 
Con ello, Derrida se refiere a una hospitalidad que se deconstruye a sí misma (Derrida, 2000, p. 5; Manzanas \& Benito, 2017, p. 4) al ser puesta en práctica como ley y no como filantropía (Kant 27) ya que al ser precisamente, ley, perpetúa una autoridad (Derrida, pp. 4-5) respecto a quién es dueño del lugar que acoge, sea este la casa o la nación. Esto constituye una aporía, definida como aquello que "solo puede ser posible en la condición de su propia imposibilidad" (traducción propia; Derrida, p. 5). Así, la hospitalidad en este contexto lleva consigo un parásito, la hostilidad (Derrida, p. 3; Manzanas \& Benito, p. 4), con quien convive en su compartida etimología, el latín hospes (Manzanas \& Benito, p. 2). Por lo tanto, son esta serie de contradicciones las que se ignoran en la articulación del discurso que acompaña al Cuadrado, y haciendo esto se simplifica e incluso infantiliza el concepto de hospitalidad. El Cuadrado es definido por Christian ante sus colegas del museo como un "acuerdo social” que "obliga a velar los unos por los otros, a ayudarnos entre nosotros”. La solemnidad de su presentación, unida al hecho de que el museo ha desembolsado alrededor de medio millón de euros en dibujar un cuadrado en el suelo, contrasta con la respuesta de su público, que corre hacia la comida cuando se anuncia que ya está lista, una de las muchas yuxtaposiciones que crean una situación absolutamente ridícula, típicas de las películas de Östlund, que resaltan las ironías de las sociedades contemporáneas. Al desdibujarse todo tipo de jerarquías dentro del Cuadrado, no hay una posición de anfitrión que acoge y otra de invitado, sugiriendo la pregunta de si realmente puede existir la hospitalidad una vez se han suspendido sus condiciones. Derrida resolverá esta cuestión modificando los términos de la hospitalidad, al sustituir la lógica de la invitación por la lógica de la visitación (Guille, 2015, p. 269-270). En otras palabras, la verdadera hospitalidad incondicionada es aquella donde no hay una invitación (que necesariamente implica un Sujeto que acoge y un Otro que es acogido) sino una visita, la cual "trae siempre aparejado un peligro o riesgo de muerte" (Guille, 2015, p. 270).

Östlund hace uso de la cinematografía para ahondar en los significados de dicha "hostipitalidad", especialmente cuando contrasta dos tipos de planos: 
el famoso plano general -donde la cámara está estática, sin música extradiegética y cuya duración suele ser larga, obligando al espectador a comprometerse con lo que ve (Westerståhl Stenport y Traylor, 2014, p. 79)y los planos subjetivos, que ofrecen el punto de vista de un personaje. Un ejemplo de ello es la conversación entre Anne, la periodista, y Christian al día siguiente de tener sexo, junto con los eventos que la preceden. Justo antes aparece otra empleada del museo para avisar a Christian de que una limpiadora ha retirado partes de una exposición creyendo que eran basura. La escena completa, pese a presentar un diálogo largo, no tiene cortes -como el plano-contraplano que podemos esperar de una escena así- ni se mueve la cámara en ningún momento, mostrando un plano general en el que Anne también aparece. Seguidamente tiene lugar la conversación entre Anne y Christian en la que esta le cuestiona detalles sobre su encuentro sexual: si suele tener sexo esporádico y qué significa para él el tenerlo. Al principio, pese a utilizarse un plano más cercano, no se hacen cortes tampoco, hasta que ambos se alejan para que no los escuche una vigilante del museo, ya que Christian, como ha demostrado en otras ocasiones, siempre se preocupa por su imagen pública. Cuando comienzan a hablar los cortes son extraños y no responden a la estructura del plano-contraplano para indicar quién habla, sino que graban las reacciones de Christian en los momentos que Anne hace las preguntas más incómodas mientras que el contraplano le corresponde a la vigilante que los mira. En los momentos en los que se enfoca a Anne, esta está callada durante incómodos silencios mientras que se escuchan los ruidos estruendosos provenientes del intento de arreglar el desastre en la exposición que había creado la limpiadora. Con la exageración de esta escena Anne, una estadounidense, parece hacer las veces de un Otro que no sigue las convenciones frías e hipócritas sobre la imagen pública por las que se rige Christian y que la vigilante parece querer descubrir. Se trata de uno de esos retos que Östlund le quiere poner a su protagonista, cuya incomodidad es evidente, para hacer tambalear los cimientos de la imagen, creada por él mismo, de director del museo que sí se muestra estable en la escena anteriormente descrita del plano general en la que habla con su compañera, 
demostrándose así que la cámara está al servicio de estas narrativas. La poca naturalidad de la cinematografía transmite perfectamente esta desestabilización y destrucción de cimientos, aún más señalada con los ruidos de demolición que acompañan la escena.

Christian, con sus actitudes ambiguas y a menudo contradictorias, personifica la delgada línea que separa la hospitalidad incondicionada del Cuadrado y la hospitalidad real y condicionada. Él, en tanto que artífice de la exhibición del Cuadrado, parece comulgar con sus valores y presentarse como esa figura que no acoge, sino que recibe una visita. Sin embargo, el peligro que entraña dicha posición se junta con las vicisitudes de su vida personal: pese a sus buenas intenciones, Christian se acoge a esa versión de la hospitalidad condicionada en la que él a menudo se posiciona como un Sujeto que, en efecto, acoge a un Otro $^{5}$ (si bien dicha acogida acabará respondiendo a los intereses personales de Christian más que a un desinteresado afán hospitalario). El mismo Östlund manifiesta que estas contradicciones eran esenciales a la hora de concebir al personaje:

Pensé en él como alguien cercano a mí, alguien que creyera en valores humanistas y que se esforzara por promoverlos -y quien al mismo tiempo, al encontrarse en determinadas situaciones de la vida real, cayera en conductas que entran en contradicción con los valores morales que defiende (traducción propia, Allard y Ciment, 2017, p.12).

Dichas contradicciones apuntan a la idea de que, en realidad, es imposible escapar de las condiciones de la hospitalidad. De hecho, lo que parece imposible de escapar es la posicionalidad del individuo, así como el espacio que cada ciudadano ocupa en una sociedad dada: ambas realidades se presentan como las condiciones mismas de la hospitalidad, como indica Derrida con su referencia a la etimología de la palabra. Dicha posicionalidad (entendida como condición existencial y trascendente, frente a la idea de

5 La idea de la "acogida al prójimo" tiene claros ecos cristianos, algo que podría parecer una conexión banal de no ser porque el propio Östlund ha manifestado que el nombre Christian evoca de manera evidente los valores cristianos (Allard y Ciment, 2017, p. 12). 
posición, inmanente y transitoria) impide a Christian ocupar el espacio neutro del Cuadrado, puesto que no puede obviar el hecho de que el lugar que ocupa en su sociedad es el de un hombre que disfruta de una posición de privilegio, representando una identidad sueca canónica a la par que internacionalista, cultivada y reputada. Östlund declara que este perfil fue deliberado: "quería que mi protagonista fuera un hombre en una situación de poder" (traducción propia, Allard y Ciment, 2017, p.12). Así, Christian cumple el estatus de sujeto deseable del que habla Lynch:

los lugares que aparecen en las películas de Östlund están permeados de un conjunto de valores económicos dominantes (centro comercial, resort de esquí, museo de arte contemporáneo), que a su vez están construidos para permitir un tipo particular de sujeto deseable, cuyo estatus depende del servicio de otros sujetos del sistema (traducción propia; 2018, p.113).

La hospitalidad, pues, se dibuja como un concepto relacional y no absoluto, que parte de las posiciones existenciales de las personas involucradas y de las relaciones entre las mismas.

Dicho concepto establece relaciones típicas de amigo/enemigo, respondiendo a un sentimiento tácito de pertenencia que aflora en situaciones de crisis. Un ejemplo claro es la escena en que Christian decide disculparse ante el niño (árabe, por cierto) al que ha acusado de robarle el móvil. Le confiesa: "tengo miedo de la gente que yo creo que vive en un edificio como el tuyo", dejando patentes sus ideas preconcebidas sobre el tipo de gente que reside en un barrio de un nivel adquisitivo mucho menor al suyo. Si bien parece que en un primer momento Christian va a hacer una reflexión sobre su propia posición privilegiada, afirmando que "esas expectativas negativas dicen mucho sobre mí", enseguida extiende los prejuicios a un ambiguo "vosotros", afirmando que "Vosotros también tendréis ideas preconcebidas sobre nosotros". Quiénes son "vosotros" y "nosotros" en el mundo de Christian es, precisamente, ese acuerdo tácito y soterrado mediante el cual ciertos individuos de la nación se (auto)presentan como huéspedes que, con suma benevolencia, acogen a aquel que llega y que esconde ese "vosotros". Para acabar de aliviar su mala conciencia, Christian le dice al niño que "Todo se 
reduce a la política y al reparto de la riqueza" y que "La sociedad tiene que arrimar el hombro", apelando a una idea abstracta de la sociedad, idea que descarga a los individuos, como él, de su responsabilidad individual. Esta idea abstracta, despojada de toda condición material, está en perfecta consonancia con la idealidad del Cuadrado y con la idea igualmente abstracta de hospitalidad que propone. En última instancia, la ayuda al Otro, que en este caso pide el niño por las consecuencias nefastas que puede tener para él el que le acusen de robo, acaba por banalizarse para convertirse en un acto de narcisismo mediante el cual las palabras de Christian no actúan como disculpa sino como un consuelo para sí mismo, como apunta Lynch: "mientras que es cierto que se siente profundamente culpable, es evidente que no está conectando con nadie sino que se limita a un círculo de reflexión narcisista donde simplemente se está dirigiendo a una imagen de sí mismo” (traducción propia; 2018, p. 106).

Tal banalización es esencial a la hora de entender la idea de hospitalidad que en última instancia vemos en The Square. Manzanas Calvo y Benito Sánchez apuntan a cómo "en las últimas décadas, los paradigmas de la hospitalidad y sus metáforas se han aplicado a formas comerciales de la hospitalidad" (traducción propia; 2017, p. 6). En efecto, tanto la actitud de Christian, que deja de lado su deber público como director de un museo por atender a sus problemas personales, como la instalación del Cuadrado parecen reforzar esta idea al representar una hospitalidad comodificada, adaptada a las necesidades del mercado o a los prejuicios individuales. Frente a la profundidad de la idea que propone el Cuadrado, la película se esfuerza en mostrar ejemplos de cómo la vida cotidiana de los personajes desecha cualquier posibilidad de una hospitalidad incondicional. La escena en que una voluntaria de alguna ONG pregunta resignada a las personas que entran a trabajar al museo por la mañana (los cuales la ignoran) si "quiere[n] salvar alguna vida" es un buen ejemplo de ello: una pregunta tan trascendente como esa pierde toda su importancia cuando es planteada en ese contexto.

Igualmente, no podemos obviar el vídeo que proponen los publicistas del museo para promocionar la instalación. En la primera reunión sobre la 
exposición, lo primero de lo que se habla es de la publicidad: hablan de vallas publicitarias y de comprar espacio en periódicos para promocionar la exposición. Esto no sorprende, puesto que ya hemos escuchado a Christian previamente hablar de su obligación de entrar a competir con otros coleccionistas privados y otros museos, con el fin de mostrar el arte adquirido a la sociedad e incluso a visitantes extranjeros. En estas declaraciones queda clara la difusa línea entre el valor social del arte y su potencial como motor económico. Por ello, la reunión orbita en torno al "potencial publicitario de cada proyecto", y según los publicistas contratados el valor de la obra reside en su capacidad de competir en el mercado. En un intento de volverse virales a toda costa, y de nuevo ejemplificando la comodificación de la hospitalidad que muestra la película, muestran en un vídeo promocional de Youtube ${ }^{6}$ a una niña sueca que vive en la calle, que explotará al meterse en el Cuadrado. Su insistencia en elegir a una niña rubia de apariencia sueca supuestamente estándar, con el objetivo de apelar al sentimentalismo del "arquetipo sueco"7 (en sus palabras), es otro ejemplo de una banal fetichización del Otro, por la que no importan las implicaciones éticas bochornosas de tal acción sino el rédito de capital -económico o cultural-que se pueda obtener de ello.

En definitiva, The Square refuerza la idea de la hospitalidad aporética derridiana, u "hostipitalidad", en tanto que plantea la naturaleza trágica y necesariamente contradictoria de la misma. No hay hospitalidad sin hostilidad, nos parecen decir tanto Derrida como Östlund. Lo que The Square nos muestra es que la imposibilidad teórica de la hospitalidad incondicional es, también, una imposibilidad más que real en las sociedades europeas contemporáneas, asoladas por el conflicto interpersonal, social y

\footnotetext{
${ }^{6}$ La presencia de YouTube en la película no es casual: Östlund ha manifestado en repetidas ocasiones su devoción por este medio por ser una ventana a diversas realidades no mediadas, presentadas de manera genuina, que muestran a las personas en situaciones comprometidas. Sus películas beben de manera directa de vídeos que encontramos en la plataforma, uno de los ejemplos más reseñable el vídeo que inspira el final de Fuerza Mayor, titulado "Idiot Spanish busdriver almost kills students" (Kelsey, 2014).

7 La elección del "arquetipo sueco" ya la hemos visto en Fuerza Mayor, donde las posiciones identitarias de la familia tradicional sueca son centrales para las implicaciones de la película.
} 
migratorio que desmiente el excepcionalismo de las comunidades europeas en que parece creer Rifkin. Östlund se consagra con esta película, así como con las que la preceden, como director genuinamente europeo al explorar y mostrar la realidad a menudo incómoda y compleja que subyace estas sociedades en ocasiones idealizadas desde fuera (como el caso del estadounidense Rikfin) o desde el punto de vista de unos autocomplacientes huéspedes.

Con un toque irónico y amargamente humorístico, las películas de Östlund presentan una Europa mucho menos comunitaria de lo que Rifkin afirma (2004, p. 90); de hecho, exhiben una descaradamente individualista. Östlund, sin embargo, no hace juicios maniqueos sobre sus personajes. En su afán antropológico los muestra como contradictorios y complejos, pero no los juzga como buenos ni malos: los poderosos en sus películas no son una caricatura que inspira desconfianza, de la misma manera que aquellos más vulnerables tampoco invitan a la empatía. La caracterización de personajes que muestran atributos desagradables como la mendiga o el niño árabe en The Square, al que Christian acusa falsamente de robo (y que tienen un precedente en Play, donde una banda de niños árabes y negros se comportan de manera similar), así como las imágenes constantes de mendigos, se yuxtaponen a los intereses de Christian por perpetuar su imagen pública y por continuar explotando económicamente la institución que dirige. Lejos de promover estereotipos racistas, Östlund intenta no caer en juicios simplistas y adherirse a su función de antropólogo, ejercida desde la sátira, con la creación de escenas incómodas, largas y absurdas que invitan a pensar en la hipocresía de la fe -e incluso admiración- en la sociedad civil más que a caer en representaciones de bondad y maldad.

Las películas de Östlund van, por tanto, más allá de la mera crítica social al cuestionar las ideas preconcebidas que circulan acerca de las supuestas comunidades ideales europeas, de las cuales Suecia es un paradigma, como él mismo explica: "para mí, la provocación debe estar dirigida a las convenciones y sobre todo a los pensamientos convencionales" (traducción propia; Allard y Ciment, 2017, p.16). Es a través de un humor grotesco e 
incómodo y de técnicas cinematográficas desconcertantes que se consigue este efecto, poniendo así el foco en las propiedades genéricas del cine. Así, aunque no se ofrezca la narrativa de los Otros, los huéspedes tampoco controlan la misma, constantemente juzgados por una cámara autónoma que parece reírse de ellos y de sus desventuras. Interpelando a la idea de Rifkin de que en la mentalidad europea los logros y avances fueron conseguidos gracias a la unión comunitaria más que al individualismo feroz del pionero fronterizo estadounidense (2004, p. 90-1), Östlund parece sugerir que es hora de que revisemos los significados de comunidad para Europa -como se sugería en la introducción con la mención al SECA- ya que la hospitalidad implícita (pero voluble) en estas nociones trae siempre consigo, como nos enseña Derrida, una hostilidad inseparable que aflora en momentos de crisis.

\section{Referencias bibliográficas}

Åberg, A. W. (2010). The Reception of Vilgot Sjöman's Curious films. En M. Larsson y A. Marklund. (Eds.), Swedish Film. An Introduction and Reader. Lund: Nordic Academic Press.

Allard, A., \& Ciment, M. (2017). Entretien avec ruben östlund: Cibler en priorite les pensées conventionnelles. Positif, (680), 11-16.

Augé, M. (1995). Non-Places. Introduction to an Anthropology of Supermodernity (J. Howe, trad.). London: Verso.

Baudrillard, J. (1998). La Sociedad de Consumo: sus Mitos, sus Estructuras (A. Bixio, trad.). Madrid: Siglo XXI.

Baudrillard, J. (1994). Simulacra and Simulation, (S. Faria Glaser, trad.). The University of Michigan Press.

Brooks, X. (2018, 11 de marzo). Ruben Östlund: 'All my films are about people trying to avoid losing face.' The Guardian. Recuperado de https://www.theguardian.com/film/2018/mar/11/ruben-ostlund-thesquare-interview-force-majeure

BUILD Series. (2018, 22 de febrero). Ruben Östlund Speaks On His Film, 'The Square' [Archivo de video]. Recuperado de https://www.youtube.com/watch?v=xVYyFExKDrU\&t=1s

Derrida, J. (2000). Hostipitality (B. Stocker y F. Morlock, trad.). Angelaki: Journal of Theoretical Humanities, 5(3), 3-18.

Dobson, K. (2017, 24 de noviembre). From Black Friday to Boxing Week: An Excerpt from Kit Dobson's Malled: Decipherin Shopping in Canada. 
National Post. Recuperado de https://nationalpost.com/entertainment/books/from-black-friday-toboxing-week-an-excerpt-from-kit-dobsons-malled-decipheringshopping-in-canada

Edholm, R. (2018). Family Framing and the Comedy of Conventions in Ruben Östlund's Force Majeure. The Nordic Journal of Aesthetics, $55-56,116-133$.

Guille, G. P. (2015). Las aporías de la hospitalidad en el pensamiento de Jacques Derrida. Eikasía: revista de filosofía, 64, 263-276.

Hjort, M. y Lindqvist, U. (2016). A Companion to Nordic Cinema. Chichester: Wiley Blackwell.

Hospitality Updated. (2017-2020). Hospitality and European Film. Universidad de Salamanca, https://hostfilm.usal.es.

Jameson, F. (1998). Postmodernism and Consumer Society. En P. Geyh, F. G. Leebron y A. Levy (Eds.), Postmodern American Fiction: a Norton Anthology. New York and London: W.W. Norton and Company: 654665 .

Jameson, F. (2002). The Dialectics of Disaster. The South Atlantic Quarterly, 101(2), 297-304.

Kant, I. (1998). Sobre la Paz Perpetua (J. Abellán, trad.). Madrid: Tecnos.

Karlsson, H. (2014). Ruben Östlund's Play (2011): Race and segregation in 'good' liberal Sweden. Journal of Scandinavian Cinema, 4(1), 43-60.

Kelsey, C. (2014, 24 de octubre). Ruben Östlund's Force of Nature. Interview Magazine. Recuperado de https://www.interviewmagazine.com/film/ruben-ostlunds-forcemajeure

Lindqvist, U. (2016). The Art of Not Telling Stories in Nordic Fiction Films. En M. Hjort y U. Lindqvist. (Eds.), A Companion to Nordic Cinema. Chichester: Wiley Blackwell.

Lynch, J. (2018). "Please Leave a Message": The Media Ecology of Ruben Östlund's Play, Force Majeure, and The Square. The Nordic Journal of Aesthetics, 55-56, 98-115.

Lyotard, JF. (1984). The Postmodern Condition: A Report on Knowledge (G. Bennington y B. Massumi, trads.). Minneapolis: University of Minnesota Press.

Manzanas Calvo, A. y Benito Sánchez, J. (2017). Hospitality in American Literature and Culture. London and New York: Routledge.

Moure, J. (2011). The Cinema as Art of the Mind: Hugo Münsterberg, First Theorist of Subjectivity in Film. En D. Chateau. (Ed.), Subjectivity: Filmic Representation and the Spectator's Experience. Amsterdam: Amsterdam UP: 23-40. 
Rifkin, J. (2004). The European Dream: How Europe's Vision of the Future is Quietly Eclipsing the American Dream. Cambridge: Polity Press.

Smallwood, C. Ruben Östlund (2015, Marzo). The Fabulist. Recuperado de https://www.aesop.com/ch/de/r/the-fabulist/ruben-ostlund

Söderbergh Widding, A. (2016). A. How to Train a Director-Film Schools in the Nordic Countries. En M. Hjort y U. Lindqvist. (Eds.), A Companion to Nordic Cinema. Chichester: Wiley Blackwell.

Westerståhl Stenport, A. y Traylor, G. (2014). Playing with art cinema? Digitality constructs in Ruben Östlund's Play. Journal of Scandinavian Cinema, 4(1), 77-84.

\section{Filmografía}

Östlund, R. (2014). Force Majeure. Suecia: Plattform Produktion / Svenska Filminstitutet / Film I Väst / Essential Filmproduktion.

Östlund, R. (2011). Play. Suecia: Plattform Produktion / Film i Väst / Société Parisienne de Production.

Östlund, R. (2017). The Square. Suecia: Plattform Produktion / arte France Cinéma / Coproduction Office. 